\title{
Cellules endocrines intestinales et pancréatiques: points très communs !
}

Il est généralement admis que les îlots pancréatiques et les cellules endocrines de l'intestin dérivent de l'intestin primitif d'origine endodermique. Situation commune à ces tissus d'importante hétérogénéité cellulaire, les facteurs responsables de la différenciation des types cellulaires sont peu connus. Des études in vivo fondées sur l'invalidation génique chez la souris, et des études fonctionnelles in vitro des facteurs de transcription impliqués dans l'expression de gènes spécifiques, conduisent très souvent à une meilleure compréhension des mécanismes de cette différenciation cellulaire. Dans le cas du pancréas, une équipe allemande vient de démontrer le rôle majeur du gène $\operatorname{Pax} 4$ sur la différenciation et la maturation des cellules pancréatiques à insuline et somatostatine, et probablement sur la fonction pancréatique exocrine [1]. Dans le cas de l'intestin, une étude américaine a démontré qu'un facteur de transcription connu pour contrôler le gène de l'insuline, le facteur BETA2, joue un rôle déterminant sur l'expression du gène codant pour une hormone intestinale, la sécrétine [2].

Un seul gène inactivé et le pancréas en est tout bouleversé! Cela était déjà vérifié par l'inactivation du gène IPF1/STF1 qui entraîne une absence de pancréas $\left(\mathrm{m} / \mathrm{s} n^{\circ} 4\right.$, vol. $\left.13, p .600\right)$ [3]. Un autre exemple est maintenant apporté par le phénotype des souris transgéniques ayant subi l'invalidation de Pax4 [1]. Le gène $P a x 4$, qui s'exprime principalement dans le pancréas, appartient à la famille des gènes murins $\operatorname{Pax}$ dont les produits sont caractérisés par des motifs fonctionnels très conservés comme l'homéodomaine ou le domaine pai- red [4]. Chez les souris $\operatorname{Pax}_{4}^{-/-}$, les altérations majeures observées concernent principalement l'absence de cellules pancréatiques de types $\beta$ (à insuline) et $\delta$ (à somatostatine). Si l'invalidation de $\operatorname{Pax} 4$ n'a aucun effet létal in utero, les souris $\mathrm{Pax}^{-/-}$ne survivent pas plus de 3 jours, présentant très tôt un retard de croissance et une déshydratation. Chez les souris normales et mutées, les cellules à insuline et à glucagon peuvent être détectées par immunohistochimie dès le jour E10,5 chez l'embryon, période qui coïncide normalement avec l'activation de Pax4 dans le pancréas. A partir du jour E16,5, chez les souris mutées uniquement, les cellules à insuline disparaissent progressivement jusqu'à la naissance. Les cellules à glucagon, en revanche, montrent une nette augmentation et s'organisent en amas. Les cellules à somatostatine dérivant de la même cellule progénitrice que la cellule $\beta$ sont, elles aussi, indétectables chez les souris mutées nouveaunées. Fait plus inattendu, la fonction pancréatique exocrine est aussi gravement affectée par la mutation génique. Rappelons que les enzymes digestives synthétisées par ce tissu sont normalement stockées dans des granules de sécrétion sous forme inactive, puis activées au niveau du duodénum au moment où le nouveau-né se met à téter. Le pancréas exocrine se vide alors de son contenu en enzymes digestives. Chez les souris $\operatorname{Pax} 4^{-/-}$de 3 jours, encore capables de téter, ces enzymes (notamment l' $\alpha$-amylase) restent anormalement abondantes dans les cellules pancréatiques exocrines. Cette situration suggère que Pax interviendrait indirectement ou directement dans le contrôle physiologique de la fonction de sécrétion enzymatique. Et si $\operatorname{Pax} 4$ contrôlait aussi la différenciation des cellules endocrines de l'intestin, lieu de synthèse et de sécrétion de la sécrétine, hormone bien connue pour son action régulatrice des sécrétions pancréatiques exocrines?

Quoi qu'il en soit, les relations pancréas-intestin endocrine se précisent de plus en plus! En effet, un facteur de transcription essentiel et déterminant pour l'expression du gène de l'insuline dans le pancréas se révèle aujourd'hui être également déterminant pour la spécificité tissulaire de l'expression du gène de la sécrétine dans l'intestin [2]. Ce facteur appelé BETA2 appartient à la famille des facteurs de transcription bHLH (basic helix-loop-helix), et a été identifié initialement sur la base de son action transactivatrice du gène de l'insuline [5].

La sécrétine est synthétisée exclusivement dans les cellules $\mathrm{S}$ entéroendocrines de l'intestin grêle de la plupart des mammifères. Le gène de la sécrétine contient une région enhancer constituée d'une séquence consensus (boîte E) liant spécifiquement les facteurs de transcription de la famille bHLH. Des études fonctionnelles in vitro ont permis de démontrer que BETA2 lie la boîte E de l'enhancer du gène de la sécrétine, et transactive ce gène. Le facteur BETA2 est d'autant plus actif qu'il s'hétérodimérise avec E47, facteur de transcription ubiquitaire transactivateur du gène de l'insuline. Un ADN antisens antiBETA2 est capable de bloquer spécifiquement l'activation d'un gène rapporteur sécrétine-luciférase dans des lignées cellulaires productrices de sécrétine. Enfin, l'expression de BETA2 seul, ou en présence de E47, 
confère à une cellule non productrice de sécrétine la capacité d'exprimer le gène rapporteur sécrétineluciférase. Des études immunohistochimiques de l'intestin de souris montrent que le facteur BETA2 est exprimé spécifiquement dans les cellules endocrines synthétisant la sécrétine, indétectable dans les autres types cellulaires intestinaux (cellules épithéliales sécrétrices, cellules à mucus, cellules de Paneth). Ces résultats indiquent clairement que le facteur de transcription BETA2, abondant dans les cellules endocrines, joue un rôle essentiel dans l'expression spécifique d'un gène codant pour une hormone intestinale dans un type cellulaire spécifique. La différenciation terminale des cellules à sécrétine est-elle sous le contrôle de BETA2 ? Les premiers résultats de l'invalidation du gène chez la souris semblent en faveur d'une telle hypothèse [2].

B.A.
1. Sosa-Pineda B, Chowdhury K, Torres M, Oliver G, Gruss P. The Pax4 gene is essential for differentiation of insulin-producing $\beta$ cells in the mammalian pancreas. Nature 1997; 386: 399-402. 2. Mutoh H, Fung BP, Naya FJ, Tsai MJ, Nishitani J, L.eiter AB. The basic helix-loop-helix transcription factor BETA2/NeuroD is expressed in mammalian enteroendocrine cells and activates secretin gene expression. Proc Natl Acad Sci USA 1997; 94 : $3560-4$.

3. Jonsson J, Carlsson L., Edlund T, Edlund H Insulin-promoter-factor 1 is required for pancreas development in mice. Nature 1994; 371 : 606-9.

4. Desplan C. Fonction des gènes Pax: synergie de liaison à l'ADN entre le domaine paired et l'homéodomaine. Med Sci 1997; 2: 147-56.

5. Naya FJ, Stellrecht CMM, Tsai MJ. Tissue-specific regulation of the insulin gene by a novel basic helix-loop-helix transcription factor. Genes Dev $1995 ; 9: 1009-19$.

\section{BRÈVES}

En absence de Pax6, point de cellule $\alpha$ pancréatique! On connaissait l'importance de $P d x l$ sur le développement du pancréas $\left(\mathrm{m} / \mathrm{s} n^{\circ}\right.$ 4, vol. 13, p. 600), et de Pax4 sur la différenciation et la maturation des cellules $\beta$ (insuline) et $\delta$ (somatostatine) pancréatiques $\left(m / s \quad n^{\circ} 8 / 9\right.$, vol. 13, p. 1063). Aujourd'hui, l'étude de l'invalidation de $\operatorname{Pax} 6$, par transgenèse chez la souris, rend ce gène responsable de la différenciation des cellules $\alpha$ sécrétrices de glucagon [1], un rôle qui s'ajoute à la participation déterminante de Pax6 dans le développement du système nerveux central, de l'œil et du nez [2]. Chez les embryons hétérozygotes pour la mutation, l'étude d'un gène marqueur permet d'observer, dès le stade 9 jours, une forte expression de Pax6 dans le pancréas et, à la naissance, une coexpression avec toutes les hormones synthétisées par les îlots de Langerhans. Les souris mutantes homozygotes, de phénotype particulièrement sévère (absence d'yeux, mort précoce), présentent au niveau pancréatique une désorganisation des îlots et une absence quasi totale de cellules $\alpha$, le tissu exocrine n'étant pas affecté. La création de souris double-transgéniques n'exprimant ni $\operatorname{Pax} 4$, ni Pax6, conduit chez les nouveau-nés à un pancréas totalement déplété de cellules endocrines, illustrant définitivement l'importance de ces deux gènes sur la formation du tissu pancréatique endocrine au cours du développement. Cette étude qui renforce une nouvelle fois l'hypothèse d'une cellule endocrine pré- curseur multipotente, permet de proposer un modèle de différenciation pancréatique endocrine: une cellule progénitrice se différencierait en cellules $\beta$ dans le cas où Pax6 et Pax4, sont tous deux exprimés, alors qu'elle ne donnerait naissance à une cellule $\alpha$ que si $\operatorname{Pax} 6$ est seul exprimé. Cela expliquerait pourquoi, chez les souris Pax4 -/-, les seules cellules pancréatiques endocrines détectées sont les cellules $\alpha$, d'ailleurs présentes de façon particulièrement abondante [3].

[1. St-Onge L, et al. Nature 1997; 387: 406-9.]

[2. Grindley JC, et al. Development $1995 ; 121$ : 1433-42.]

[3. Sosa-Pineda B, et al. Nature 1997 ; 386: 399-402.] 


\section{BRÈVES}

L'homologue humain du facteur de transcription achaete-scute (hASHl) de la drosophile joue un rôle essentiel dans la différenciation des cellules neuroendocriniennes pulmonaires normales et tumorales. La protéine ASH1 (achaete-scute homologue) fait partie d'une famille de facteurs de transcription bHLH (basic helix-loop-helix) jouant un rôle important dans la différenciation neuronale chez la drosophile et les vertébrés [1]. Durant le développement embryonnaire chez la souris, la distribution des cellules synthétisant ASH1 est similaire à celle des cellules pulmonaires neuroendocriniennes. De même, la synthèse de l'analogue humain, hASH1, dans différentes lignées cellulaires de cancers pulmonaires est corrélée à la présence d'un phénotype neuroendocrinien [2]. A l'inverse, certains variants de ces lignées cellulaires ayant perdu leurs caractéristiques neuroendocriniennes ne synthétisent plus hASHl. En particulier, l'expression d'un mutant oncogénique de H-RAS dans une lignée de cancer pulmonaire à petites cellules (NCI-H249) entraîne la perte du caractère neuroendocrinien et de l'expression du gène hASH1. L'équipe de Douglas Ball à Baltimore (MD, USA) rapporte maintenant que la délétion par recombinaison homologue du gène ASH1 chez la souris empêche tout développement des cellules neuroendocriniennes pulmonaires [3]. Chez les souris homozygotes $\mathrm{ASH} \mathrm{H}^{-/-}$, différents marqueurs de la différenciation neuronale comme le CGRP ( $\mathrm{cal}$ citonin gene related peptide), la NSE (neuron specific enolase) ou la chromogranine ne sont plus mis en évidence au niveau pulmonaire. De même, un traitement par oligonucléotides antisens de $h A S H 1$ des lignées cellulaires de cancer pulmonaire à petites cellules DMS-53 et NCI-H209 entraîne la perte d'expression de ces mêmes marqueurs de différenciation neuroendocrinienne. Le facteur de transcription hASHl participerait donc au développement normal des cellules neuroendocriniennes pulmonaires et aussi à l'acquisition du phénotype neuroendocrinien des redoutables cancers pulmonaires à petites cellules.

[1. Ghysen A, Dambly-Chaudière C. Med Sci 1995; 11 : 178-88.]

[2. Ball DW, et al. Proc Natl Acad Sci USA 1993; 90 : 5648-52.]

[3. Borges M, et al. Nature 1997; $386: 852-5$.]

\section{Cours de Biologie Moléculaire} de la Cellule

Enseignement Pratique

9 mars-10 avril 1998

Ce cours, conjointement organisé par l'Institut PasCe cours, conjointement organise par 9 mars au 10 avril 1998 a plein temps, à 'Institut Pasteur à 10 avril 1998 à plein temps, à Institut Pasteur à
Paris. II est destine à des chercheurs du secteur public et privé, ayant une formation des facultés de sciences, de médecine, de pharmacie ou des écoles vétérinaires. Les candidats doivent avoir une bonne connaissance, niveau maîtrise, en biologie moléculaire. Les techniques de base de biologie moléculaire ne seront pas enseignées (exemple: clonage, séquencage de gènes, etcl. Ce cours donne liev à un diplome de Institut Pasteur suite à un examen qui se déroulera à la fin du mois d'avril.

Le thème central de ce cours concerne l'étude de la Le theme central de ce cours concerne letude de la
cellule eucaryote. Cet enseignement est très orienté vers l'initiation expérimentale, et fera une large place aux nouvelles, techniques ainsi qu'á la démarche scientifique actuelle pour l'étude des fonctions cellulaires. Les travaux pratiques seront accompagnés de conférences theoriques sur les thèmes suivants:

- Organisation fonctionnelle de la cellule : compar timents membranaires, cytosquelette, polarité celulaire

- Les routages intracellulaires : transport des protéines membranaires et sécrétées, endocyłose des macromolécules

- Les contacts et la communication entre cellules

- La différenciation cellulaire

- La signalisation et la transduction des messagers Laires

- Le cycle cellulaire.

Les techniques mises en œuvre seront celles de I'analyse génétique, la transfection ef l'expression de gènes clonés la culture cellulaire, la reconstitution in vitro des fonctions cellulaires, la visualisation des constituants cellulaires y compris par les techniques les plus récentes de microscopie confocale et niques les plus
d'imagerie.

Avec la participation de : S. Amigorena, M. Arpin, Ch. Babinet, M. Bornens, F. Colbère-Garapin, P. Cossart, E. Coudrier, F. Dautry, A. Dautry-VarP. Cossart, E. Coudrier, F. Dautry, A. Dautry-Varsat,' Hollock, C. Hopkins, A Hubbard, E Karsenti' O. Kellerman, F. Képés, P. Legrain, D. Louvard, O. Kellerman, F. Képes, P. Legrain, D. Louvard, $M$. Weiss. Les cours théoriques seront assurés par des enseignants français et européns.

Responsables du cours : A. Dautry-Varsat et D. Louvard

Renseignements et Inscriptions, date limite le jer décembre 1997

Mme Banisso

Secrétariat des Enseignements et des Stages

Institut Pasteur, 28, rue du Docteur-Roux, 75724 Paris Cedex 15, France

Tél. : 0145688141 ou 0140613362 Fax : 0140613046

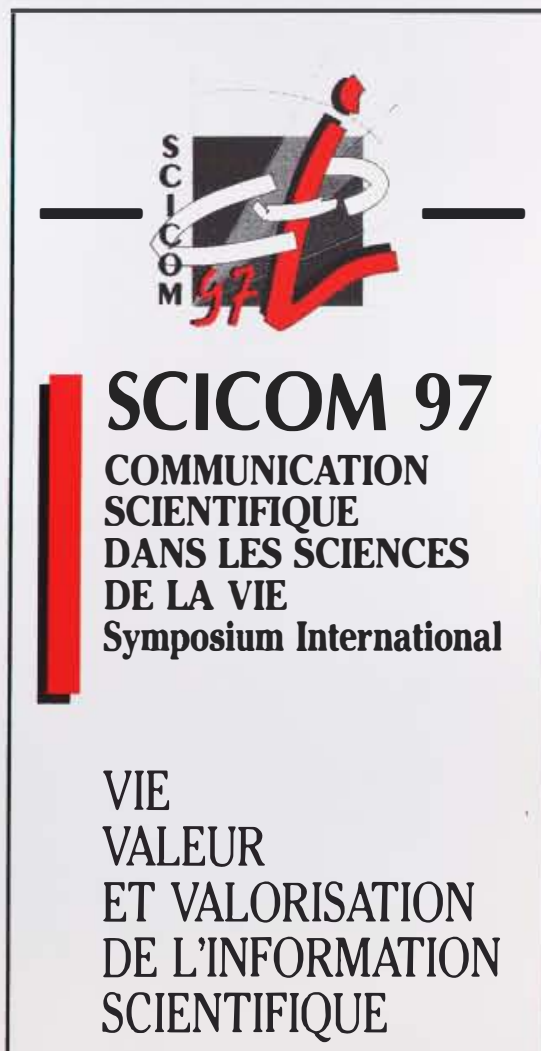

17-19 septembre 1997

\section{Faculté de Médecine de Nancy - France}

\section{SOUTIENS \\ GRIP}

Groupe de Recherche

en Immunopathologie, AIM

Association pour les applications de l'Informatique à la Médecine.

Ministère de l'Enseignement Supérieur

et de la Recherche, Université Henri-Poincaré Nancy I
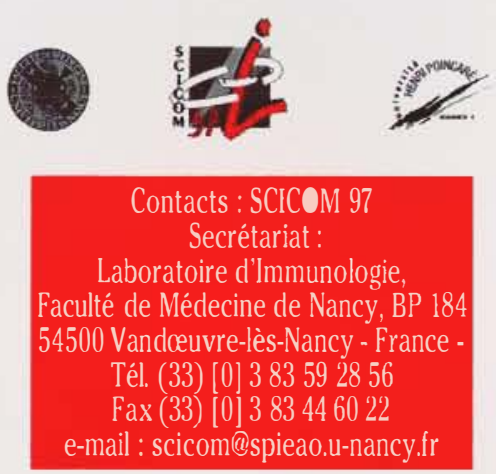